\title{
3. \\ Class II histone deacetylases: versatile regulators
}

\author{
Eric Verdin, Franck Dequiedt and Herbert G. Kasler \\ Gladstone Institute of Virology and Immunology, University of California San Francisco, PO Box 419100, San Francisco, \\ CA 94141, USA
}

\begin{abstract}
Histone acetylation and deacetylation play essential roles in modifying chromatin structure and regulating gene expression in eukaryotes. Histone deacetylases (HDACs) catalyze the deacetylation of lysine residues in the histone $\mathrm{N}$-terminal tails and are found in large multiprotein complexes with transcriptional co-repressors. Human HDACs are grouped into three classes based on their similarity to known yeast factors: class I HDACs are similar to the yeast transcriptional repressor yRPD3, class II HDACs to yHDA1 and class III HDACs to ySIR2. In this review, we focus on the biology of class II HDACs. These newly discovered enzymes have been implicated as global regulators of gene expression during cell differentiation and development. We discuss their emerging biological functions and the molecular mechanisms by which they are regulated.
\end{abstract}

The DNA of eukaryotic cells in chromatin is tightly wrapped around octamers of histone proteins, restricting its accessibility to factors involved in DNA replication and transcription. Local or extended structural changes in chromatin play an important role in the control of gene expression and are governed by complexes that remodel chromatin and by enzymes that posttranslationally modify histones (reviewed in Ref. [1]).

All core histone proteins are reversibly and dynamically acetylated at multiple sites in their $\mathrm{N}$-terminal tails. Hyperacetylated histones are generally found in transcriptionally active genes and hypoacetylated histones in transcriptionally silent regions, such as heterochromatin. The level of histone acetylation at a particular locus in chromatin reflects the competing activities of histone acetyltransferases and histone deacetylases (HDACs). The identification and characterization of numerous transcriptional regulators possessing histone acetyltransferase or HDAC activities has validated the prediction that histone acetylation plays a critical role in transcriptional regulatory mechanisms (for general reviews see Ref. [2]).

Eighteen distinct human HDACs are grouped into three classes based on their primary homology to three Saccharomyces cerevisiae HDACs. Class I HDACs (HDAC1, -2, -3, -8 and -11) are homologous to yRPD3, share a compact structure, and are predominantly nuclear proteins expressed in most tissues and cell lines (Fig. 1a) (reviewed in Ref. [3]). Class II HDACs, the subject of this

Corresponding author: Eric Verdin (everdin@gladstone.ucsf.edu). review, are homologous to yHDA1 and are subdivided into two subclasses, IIa (HDAC4, $-5,-7$ and -9 and its splice variant MITR) and IIb (HDAC6 and HDAC10), based on sequence homology and domain organization (Fig. 1a,b) [4-12]. Whereas class I and II HDACs and their

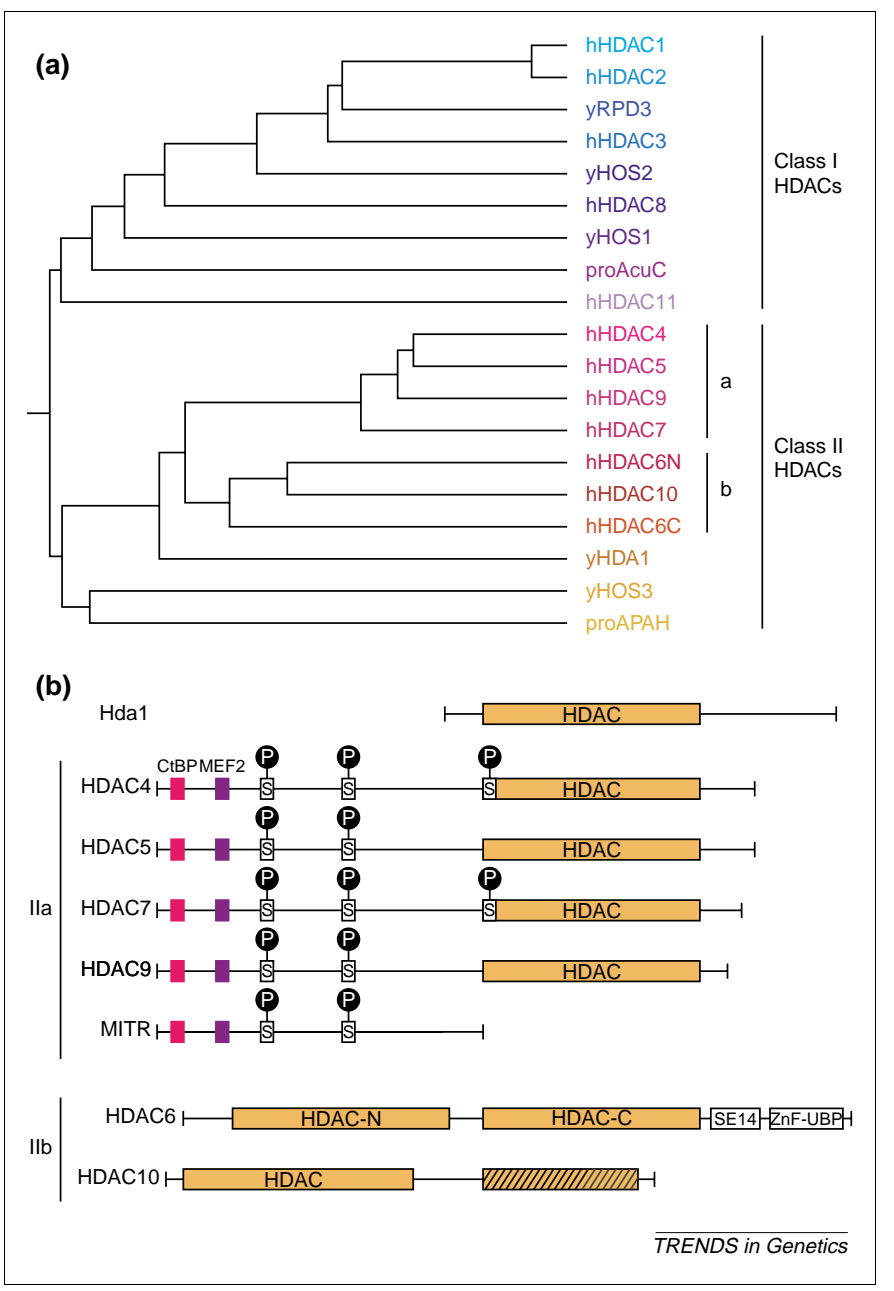

Fig. 1. Organization of class II histone deacetylases (HDACs) (a) Phylogenic tree analysis of known mammalian HDACs and their yeast orthologs for the HDAC class I and II families (adapted from Ref. [11]). The human proteins are preceded by $h$, the yeast proteins by $y$ and the prokaryotic proteins by pro. (b) Schematic structure of class II HDACs. The class Ila HDACs, HDAC4, $-5,-7$ and -9 , and MITR (an HDAC9 splice variant), share an N-terminal domain of $\sim 450-600$ amino acids. Domains in this region mediate interactions with the transcriptional co-repressor CtBP, the MEF2 family of transcription factors, and others (see Fig. 2 for further details). Conserved phosphorylation sites that play a crucial role in nuclear/ cytoplasmic shuttling are indicated. The class Ilb HDACs, HDAC6 and HDAC10, are characterized by the presence of two tandemly arranged HDAC domains (the second domain is truncated in the case of HDAC10). 


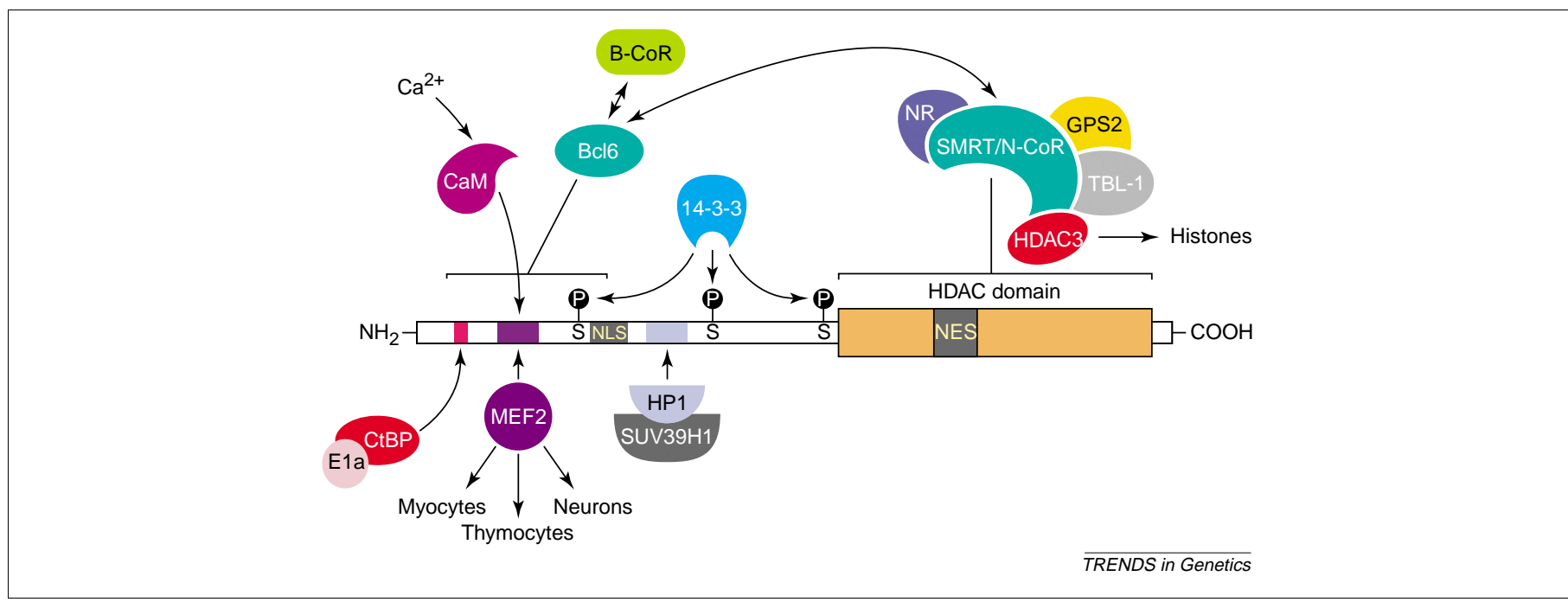

Fig. 2. Interaction partners of class lla histone deacetylases (HDACs). Class Ila HDACs interact with several partners through distinct domains. The N-terminal domain is used primarily as a targeting domain for distinct promoters by the MEF2 transcription factors. Interaction of class Ila HDACs with HP1, CtBP and the SMRT/N-CoR and B-CoR co-repressor complexes mediates the transcriptional repressive activities of class II HDACs.

S. cerevisiae orthologs, yRPD3 and yHDA1, all share some degree of homology in their catalytic domain, class III HDACs are homologous to ySIR2 and show no homology to class I and II proteins.

\section{The class Ila HDACs: HDAC4, $-5,-7$ and -9}

HDAC4, -5, -7 and -9 contain a highly conserved C-terminal catalytic domain $(\sim 420$ amino acids) homologous to yHDA1 and an N-terminal domain with no similarity to other proteins (Fig. 1b) [4-7,13]. MITR, a splice variant of HDAC9, consists of only its N-terminus without an HDAC domain. The activity of the class IIa HDACs is regulated at several levels, including tissuespecific gene expression, recruitment of distinct cofactors and nucleocytoplasmic shuttling.

\section{Tissue-specific expression}

Whereas most class I HDACs are ubiquitously expressed, the class IIa HDACs are expressed in a restricted number of cell types. Three of the class IIa HDACs, HDAC4, -5 and -9 , show highest expression in heart, skeletal muscle and brain, where their biological activities might be partially redundant $[4,5,7,13-15]$. In contrast to initial reports describing highest HDAC7 expression in heart and lung tissues $[16,17]$, we have recently observed that HDAC7 is most highly expressed in CD4/CD8 doublepositive thymocytes (F. Dequiedt et al., unpublished).

\section{Interaction partners}

HDACs represent the catalytic components of large multiprotein complexes. They do not bind directly to DNA and are thought to be recruited to specific promoters through their interaction with DNA sequence-specific transcription factors (Fig. 2). The MEF2 family of transcription factors is one of the major targets of class IIa HDACs and will be discussed in detail below (see section on the biological role of HDACs). Other interactions occur with CtBP (E1A C-terminal binding protein), 14-3-3 proteins, calmodulin $(\mathrm{CaM})$, transcriptional co-repressors, heterochromatin protein $\mathrm{HP} 1 \alpha$ and SUMO.
CtBP1 was originally identified as an adenovirus E1A interacting protein and later found to interact with several Drosophila DNA-binding proteins, including Hairy, Snail, Krüppel and mammalian basic Krüppel-like factor 3 (BKLF/KLF3) (reviewed in Ref. [18]). CtBP associates with each of these proteins, as well as with HDAC4 and -5 and MITR, through a PXDLS motif, and acts as a transcriptional repressor [19]. However, since CtBP can still repress transcription in the absence of HDAC activity, the precise mechanism of CtBP-mediated transcriptional repression remains unclear [18].

The class IIa HDACs all interact with 14-3-3 proteins [20-24]. This interaction plays a critical role in the nucleocytoplasmic shuttling of class II HDACs (see section on dynamic subcellular localization for an in depth discussion of the role of 14-3-3 proteins).

The MEF2-binding domain of HDAC4 shares some features with known CaM-binding domains [25]. HDAC4 binds to CaM in vitro, and binding of CaM disrupts the MEF2-HDAC4 interaction. This mechanism could contribute to the $\mathrm{Ca}^{2+}$-dependent activation of MEF2 [26]; however, the significance of these observations for the biology of class IIa HDACs remains to be assessed.

Class II HDACs interact with two closely related co-repressors, SMRT (silencing mediator for retinoid and thyroid receptors) and $\mathrm{N}-\mathrm{CoR}$ (nuclear receptor co-repressor). This interaction is critical for the enzymatic activity of class IIa HDACs and is discussed separately below. In addition, the $\mathrm{N}$-terminal $\mathrm{BTB} / \mathrm{POZ}$ domain of BCL6, a sequence-specific transcriptional repressor with Krüppel-like zinc-finger motifs that is involved in the pathogenesis of non-Hodgkin's B-cell lymphomas [27,28], can bind either to SMRT, leading to the recruitment of the SMRT/N-CoR co-repressor complex [29-31], or to B-CoR, another co-repressor protein [32]. Therefore, class IIa HDACs might modulate the transcriptional repression of BCL6 and participate in its role in B-cell activation and differentiation, inflammation, and cell-cycle regulation [33]. BCL6 also interacts directly with a conserved region in the N-terminal domain of class IIa HDACs [34] (Fig. 2). 
Thus, class IIa HDACs can be recruited to Bcl-6-regulated promoters by two mechanisms: direct interaction with BCL6 or indirect recruitment through co-repressors such as SMRT/N-CoR and B-CoR. It is not clear why B-CoR and BCL6 interact with such a disparate array of class I and class II HDACs. Further experiments are needed to assess the biological relevance of these observations.

HDAC4 and -5 and MITR interact with HP1 $\alpha$. The interaction is mediated by an $\mathrm{N}$-terminal domain that is distinct from other interacting sites on class II HDACs (Fig. 2). HP1 contains a chromodomain involved in the specific recognition of methylated lysine 9 of histone. HP1 also interacts with the histone methyltransferase SUV39H1. Since histone H3 must be deacetylated before it can be methylated, these observations suggest that the class II HDAC-HP1 interaction could be important in the establishment and maintenance of heterochromatin [35].

Finally, HDAC4, MITR and HDAC6 associate with and become modified by SUMO-1, a ubiquitin-like protein $[36,37]$. The biological significance of this modification has not been established.

\section{Dynamic subcellular localization}

All class IIa HDACs shuttle between the nucleus and the cytoplasm [6,20,21,23,26,38-40]. Class IIa HDACs bind to $14-3-3$ proteins, a family of highly conserved acidic proteins (reviewed in Ref. [41]). This binding is dependent on the phosphorylation of two or three conserved $\mathrm{N}$-terminal serine residues in class IIa HDACs and mediates their cytoplasmic sequestration (Fig. 1b) [20,21,23]; mutation of these sites prevents the export of class IIa HDACs from the nucleus to the cytoplasm [20-24,40,42] (Fig. 1b). The sequences of the phosphorylation sites in class IIa HDACs are closely related to consensus phosphorylation sites for $\mathrm{Ca}^{2+} / \mathrm{CaM}$-dependent protein kinases (CaMKs) [43], and CaMK-mediated phosphorylation of HDAC4, $-5,-7$ and -9 promotes their association with 14-3-3 proteins and stimulates their nuclear export to the cytoplasm [21-24,44]. Recent experiments in cardiomyocytes indicate that an unidentified HDAC kinase targets the same phosphorylation sites in class IIa HDACs in response to hypertrophic signals [45].

It is thought that 14-3-3 proteins modify the subcellular localization of targets by interfering with nuclear import and export signals. A nuclear import signal containing an arginine/lysine-rich motif has been mapped in HDAC4 (amino acids 251-272) and -5 (amino acids 264-285) $[26,46]$, and appears to be conserved in HDAC7 and MITR/HDAC9. Phosphorylation-dependent binding of 14-3-3 proteins to the N-termini of class II HDACs masks the nuclear import signal and prevents nuclear import. A CRM1-dependent nuclear export signal has been mapped in HDAC4 (amino acids 1056-1069) and HDAC5 (amino acids 1086-1099) [44,46]. This nuclear signal becomes active upon binding of 14-3-3 proteins to the N-terminus of class II HDACs. These observations suggest that phosphorylation of the $\mathrm{N}$-terminus of class IIa HDACs and/or recruitment of 14-3-3 proteins induces a longdistance conformational change that unmasks a latent nuclear export signal in their C-terminus.

The nucleocytoplasmic shuttling of class II HDACs regulates their activities as transcriptional repressor proteins. Overexpression of constitutively active CaMKs or signal-dependent activation of CaMKs induces the relocalization of class IIa HDACs to the cytoplasm and suppresses their repressive activity $[22,47]$. By contrast, mutation of the phosphorylation sites of class IIa HDACs abolishes their cytoplasmic export and enhances their repressive effects during muscle differentiation and T-cell apoptosis [40,43]. Cytoplasmic relocalization of class II HDACs removes these enzymes from their substrates the histone proteins in chromatin - and dissociates them from the SMRT/N-CoR-HDAC3 complex, leading to their enzymatic inactivation, as discussed in the next section $[16,48]$.

\section{Regulation of HDAC catalytic activity}

Class IIa HDACs interact directly with SMRT and N-CoR through their C-terminal HDAC domains [17,49,50]. Both SMRT and N-CoR interact with unbound nuclear receptors and with a growing list of other transcription factors (reviewed in Ref. [51]). In addition, both SMRT and N-CoR participate in the formation of a stable multiprotein complex consisting of HDAC3, transducin ( $\beta$ )-like 1 (TBL1) $[52,53]$ and GPS2, a protein involved in intracellular signaling [54]. A recent proteomic analysis of the components of the $S$. cerevisiae SET3 complex showed the presence of two HDACs, Hos2 and Hst1 [55]. Hos2 is related to the yeast Hda1 deacetylase, while Hst1 is a Sir2-like protein with NAD-dependent deacetylase activity. The SET3 complex also contains a WD40 protein with homology to TBL1, suggesting that this complex is a yeast ortholog of the SMRT/NCoR complex. It is not clear at this point why multiple HDACs from different classes are assembled in a single multiprotein complex. Distinct enzymes might confer unique substrate specificities to the complex. The dependency of class III enzymes on $\mathrm{NAD}^{+}$ could also link the activity of the complex to the metabolic status of the cell. A similar combination of class II and class III enzymes in a multiprotein complex was recently observed for HDAC6 and SIRT2, a human class III HDAC [56].

SMRT and N-CoR interact both with class IIa HDACs and with HDAC3 through distinct domains [52,53,57,58], an observation that explains why class IIa HDACs coimmunoprecipitate with HDAC3 $[5,16,48]$. Class IIa HDACs are enzymatically inactive alone as purified recombinant proteins. However, they are associated with enzymatic activity when bound to the SMRT/N-CoR-HDAC3 complex $[16,48]$. HDAC3 is also catalytically inactive alone as purified protein but becomes enzymatically active when bound to SMRT/N-CoR, even in the absence of a class IIa HDAC [48,58]. By contrast, class IIa HDACs alone are still enzymatically inactive after binding to the SMRT/N-CoR proteins in vitro.

This evidence is compatible with two models. First, class IIa HDACs might not be functional HDACs in the context of the SMRT/N-CoR complex but might serve to recruit pre-existing, enzymatically active SMRT/N-CoR complexes that contain HDAC3. In this model, the SMRT/N-CoR co-repressors provide a structural link between active HDAC3 and inactive class IIa HDACs. In 
a second model, class II HDACs might become activated only in the presence of HDAC3. There is extensive evidence for HDAC-HDAC interactions: the solved structure of Rpd3 shows a dimer, HDAC1 and -2 are found in the same multiprotein complex, and HDAC6 contains two tandemly arranged HDAC domains. These interactions might indicate that HDAC-HDAC interactions are important for the regulation of HDAC activity.

Interestingly, we have noted that several mutations in amino acids that form the catalytic pocket of class IIa HDACs also result in a loss of interaction with SMRT/N-CoR. These observations suggest the existence of interactions between amino acids in the catalytic center of class IIa HDACs and the surface of these proteins. Such interactions could regulate the enzymatic activity of the enzyme as a function of its interactions with other proteins and could ensure that the HDACs only become enzymatically active after incorporation in the appropriate multiprotein complex.

\section{Biological roles of class Ila HDACs}

The many interactions between class IIa HDACs and transcriptional regulators suggest a wide variety of potential biological roles. However, most of these interactions have not been examined in a biological context. By contrast, the importance of interactions between MEF2 and class IIa HDACs has been demonstrated in several tissue culture and animal models. MEF2 plays a significant transcriptional regulatory role in myogenesis, in negative selection of developing thymocytes, and in the transcriptional regulation of Epstein-Barr virus (EBV) (for a complete review see Ref. [59]). The striking similarity of MEF2 regulation in neurons and its role in neuronal resistance to excitotoxicity suggests that class IIa HDACs also play a significant role in neurons [60-62].

Class IIa HDACs inhibit myogenesis by binding to MEF2 at several promoters critical for the muscle differentiation program (for recent reviews see Refs $[63,64]$ ). HDAC4, $-5,-7$ and -9 interact with MEF2 proteins through a highly conserved 17 amino acid motif located in their N-termini $[7,23,38,65,66]$. Expression of an HDAC-VP16 fusion protein, in which the VP16 activation domain replaces the catalytic domain of HDAC4 or -5 , enhances myogenic conversion [47]. Activation of the CaMK signaling pathway also overcomes the HDAC-mediated repression of muscle-specific gene expression and induces the myogenic conversion program [22,64]. Further evidence for the role of class IIa HDACs in cardiac and skeletal myogenesis comes from the phenotype of HDAC9-deficient animals. A phosphorylation mutant of HDAC9 in the CaMK IV sites inhibits the myogenic program [24]. Conversely, HDAC9-deficient mice show a striking susceptibility to stress-induced cardiac hypertrophy [45]. In cardiomyocytes, phosphorylation mutants of HDAC5 or MITR block the induction of hypertrophy-associated genes in response to phenylephrine [45]. These observations suggest that class IIa HDACs are master regulators of myocyte development. Bound to MEF2 proteins in the promoters of multiple genes, they play a critical role in repressing these genes until the appropriate myogenic differentiation signal is delivered.
In the thymus, developing CD4/CD8 double-positive $\mathrm{T}$ cells that receive a strong signal from major histocompatibility complex (MHC)-self-peptide through their antigen receptors are deleted by an apoptotic process termed negative selection. The apoptotic process is activated by the expression of Nur77, an orphan steroid receptor [67-69]. Constitutive expression of Nur77 in thymocytes results in a dramatic involution of the thymus, whereas expression of a dominant-negative Nur77 interferes with negative selection [70,71]. The expression of Nur77 in response to antigen receptor signals is tightly controlled through two MEF2-binding sites in the Nur77 promoter (Fig. 3) [72]. As in other systems, MEF2-dependent transcription in $\mathrm{T}$ cells is activated by $\mathrm{Ca}^{2+}$ signals and by ectopic expression of activated CaMK IV, calcineurin, or ERK5/BMK1 [73-75].

A MEF2-family protein, MEF2-D, is expressed in thymocytes and associates with both HDAC4 and Cabin-1 under basal conditions in T-cell lines (Fig. 3) [25,74,76,77]. Activation of T-cell receptors disrupts the interaction of MEF2-D with HDAC4 and Cabin-1, most probably through a $\mathrm{Ca}^{2+}$ signal and CaM activation. HDAC7 is highly expressed in CD4/CD8 double-positive thymocytes, which undergo positive or negative selection (Fig. 3) (F. Dequiedt et al., unpublished). HDAC7 also associates with MEF2-D in CD4/CD8 double-positive T cells, becomes dissociated from MEF2-D during T-cell activation and shuttles out of the nucleus. The nuclear export of HDAC7 in thymocytes is dependent on three serine residues in the $\mathrm{N}$-terminus of the protein, as described above for other class IIa HDACs. HDAC7 overexpression suppresses Nur77 induction and thymocyte apoptosis in response to activation of T-cell receptors. Conversely, expression of an HDAC7-VP16 fusion activates Nur77 expression in the absence of such activation, and knockdown of HDAC7 through RNA interference leads to increased apoptosis in response to T-cell receptor activation. These findings, combined with the recent finding that deletion of the MEF2interacting domain of Cabin-1 results in no detectable phenotype with respect to thymic negative selection [78], suggest that HDAC7 plays a critical role in the repression of Nur77 during thymic maturation of T cells. Studies are currently under way to validate these observations in transgenic and knockout mice.

EBV is a human herpesvirus capable of latently infecting $B$ cells. The latent virus can be reactivated by crosslinking of the B-cell antigen receptor [79,80]. Reactivation of latent EBV proceeds through three MEF2-binding sites in the promoter for the immediate-early gene BZLF1, which encodes the transcriptional activator ZEBRA. MEF2-dependent transcription of BZLF1 is activated by $\mathrm{Ca}^{2+}$ flux; a similar mechanism governs transcription of the Nur77 promoter in T cells [81]. In a B-cell line carrying latent EBV, transfection of HDAC4- or HDAC5-VP16 fusions reactivates viral expression [82]. These results indicate that EBV latency, like myogenesis and Nur77 expression, is regulated by a $\mathrm{Ca}^{2+}$-dependent MEF2 switch in which class IIa HDACs mediate basal repression. 


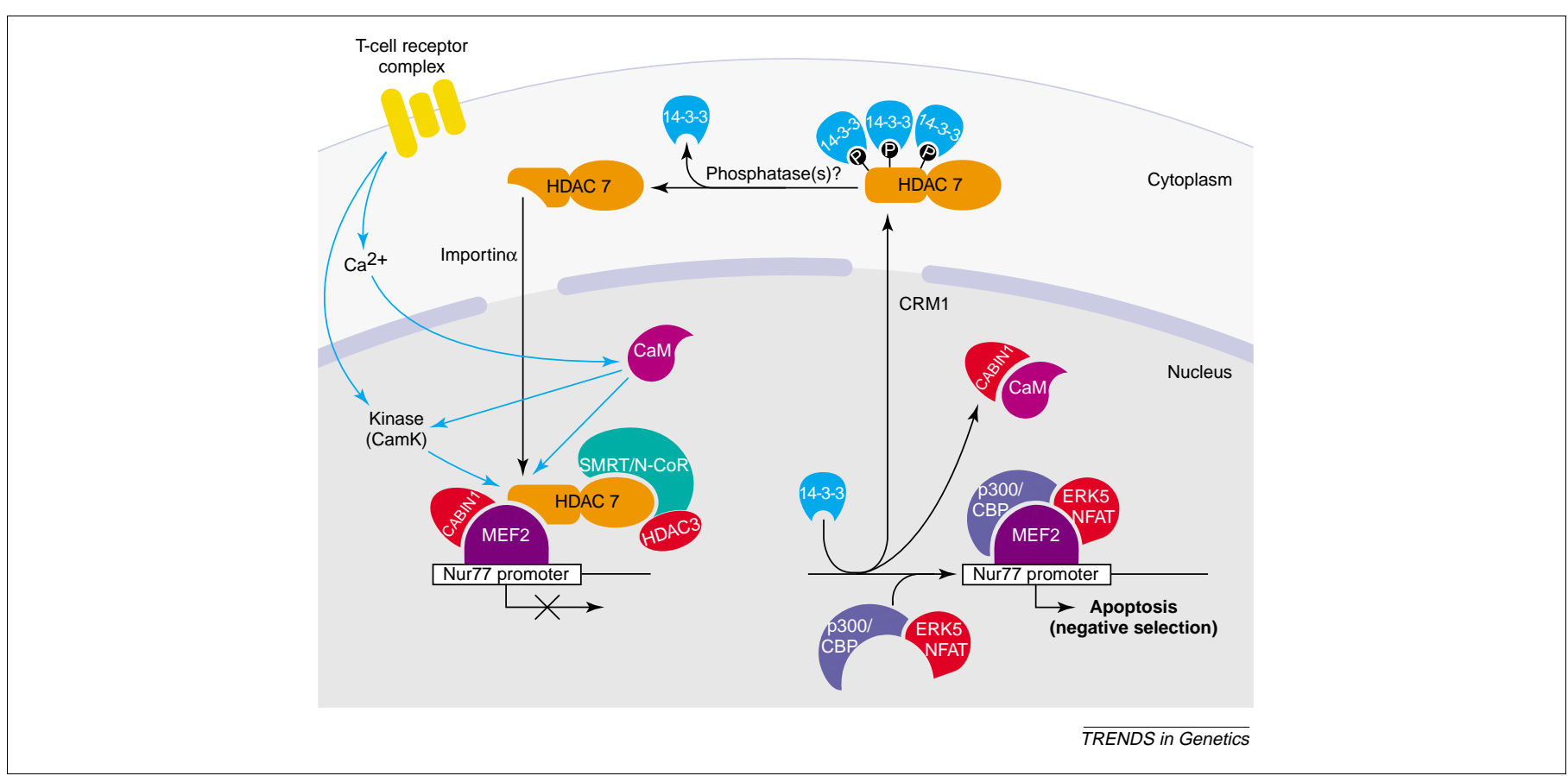

Fig. 3. Regulation of Nur77 promoter activity in developing thymic T cells by class II histone deacetylases (HDACs). Class II HDACs, primarily HDAC7 in the thymus, repress the latent activating potential of MEF2 transcription factors, both by preventing the binding of coactivators and by direct enzymatic activities associated with class lla HDACs. Elevation of intracellular $\mathrm{Ca}^{2+}$ levels, and possibly of kinases, through activation of the T-cell receptor in turn activates the Ca ${ }^{2+}$ sensor calmodulin (CaM), which can directly displace class II HDACs from MEF2. In addition, CaM-dependent activation of $\mathrm{Ca}^{2+} / \mathrm{CaM}$-dependent protein kinases (CaMK) I and II results in the phosphorylation of the N-terminal domain of class II HDACs. Phosphorylated class Ila HDACs are displaced from MEF2, most probably because of conformational changes in the interacting region. The 14-3-3 proteins bind to phosphorylated class II HDACs and mediate CRM1-dependent nuclear export, allowing the sustained expression of MEF2 target genes. For activation of transcription, coactivators (p300/CBP) and activators (NF-AT, ERK5) are recruited to the promoter. Unknown cytoplasmic phosphatases dephosphorylate class II HDACs, leading to their release from 14-3-3 proteins.

\section{The class Ilb HDACs: HDAC6 and HDAC10}

Class IIb HDACs are characterized by duplicated HDAC domains, although this duplication is partial in the case of HDAC10 (Fig. 1b). Class IIb HDACs also show some degree of tissue-specific gene expression: HDAC6 is predominantly expressed in testis $[13,83]$, and HDAC10 is expressed in liver, spleen and kidney $[8,10,12]$. Different splice variants are observed for HDAC10, as shown for HDAC3, -7 and -9 , suggesting an additional level of regulation by RNA processing. Analysis of the catalytic activity of the two separate HDAC domains of HDAC6 by sitedirected mutagenesis suggested that the two domains might function independently [5]; however, separation of the two domains results in loss of enzymatic activity (W. Fischle and E. Verdin, unpublished). In HDAC10, the C-terminal catalytic domain lacks the active pocket residues required for enzymatic activity. The enzymatic activities of HDAC6 and HDAC10 are more resistant to trapoxin and sodium butyrate than those of class I and class IIa HDACs $[9,84]$. Deletion of the second incomplete catalytic domain of HDAC10 restores its sensitivity to both sodium butyrate and trapoxin, suggesting that the two HDAC domains functionally interact [9].

HDAC6 shuttles in and out of the cell nucleus. In the absence of a stimulus, the majority of HDAC6 is localized to the cytoplasm, but cell-cycle arrest is associated with the partial translocation of the protein to the cell nucleus [85]. HDAC10 is also primarily cytoplasmic but shows significant nuclear staining in several cell lines [8-10,12]. The cytoplasmic location of both HDAC6 and HDAC10, unlike that of class IIa HDACs, is insensitive to leptomycin B
$[8,9,85]$. The subcellular localization of HDAC6 is dependent on a strong nuclear export signal (NES1) in the $\mathrm{N}$-terminus of the protein [85]. Several putative export sequences were identified in HDAC10 [8,12], but it is not known whether they function as true export signals.

HDAC6 is part of a multiprotein complex that includes two mammalian orthologs of yeast proteins involved in the control of protein ubiquitination: p97/VCP, a mammalian homolog of yeast Cdc48p, and phospholipase A2 activating protein, a homolog of yeast UFD3 (ubiquitin fusion degradation protein 3) (Fig. 4) [83]. HDAC6 contains a C-terminal zinc finger that shows significant homology to a zinc finger located in several ubiquitin-specific proteases (ZnF-UBP) [83] (Fig. 4). The ZnF-UBP motif (also identified as a polyubiquitin-associated zinc finger or PAZ domain) [86] mediates the binding of ubiquitin to HDAC6 $[83,86]$. The specific interaction of proteins involved in protein ubiquitination and the fact that both acetylation and ubiquitination target lysine residues suggest that HDAC6 could participate in the deacetylation of proteins before their ubiquitination.

Cytoplasmic HDAC6 shows striking co-localization with the microtubule network $[87,88]$. Acetylation of $\alpha$-tubulin, at lysine 40 , has been used as a marker of microtubule stability [89]. HDAC6 functions as a specific tubulin deacetylase in vivo, and purified HDAC6 deacetylates $\alpha$-tubulin in assembled microtubules in vitro $[87,88]$. HDAC6 overexpression promotes chemotactic cell movement, a cellular function dependent on microtubules. We have independently observed that SIRT2, a $\mathrm{NAD}^{+}$-dependent class III HDAC, deacetylates lysine 40 of 


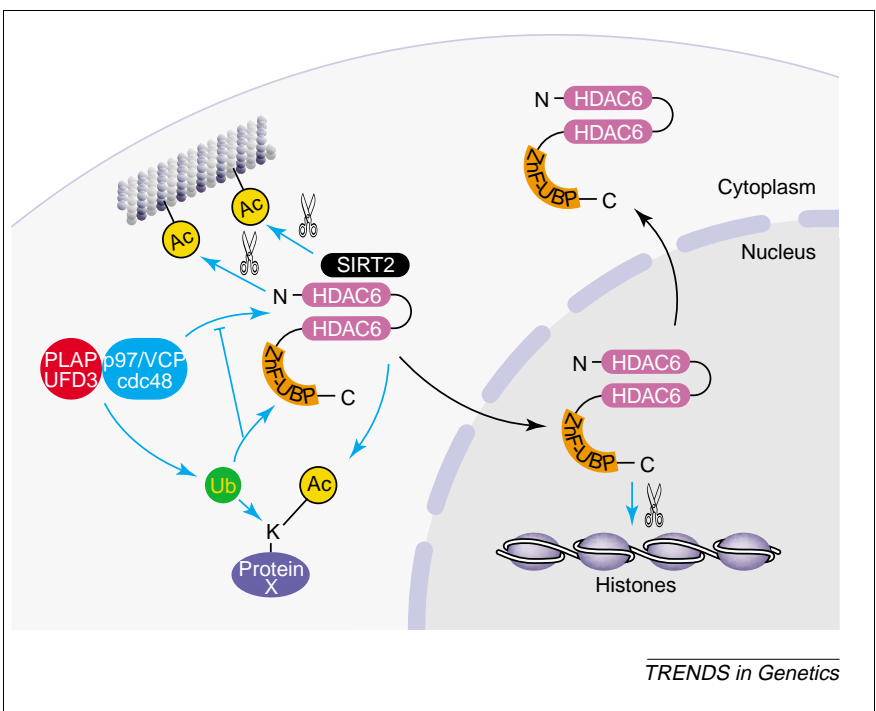

Fig. 4. Histone deacetylase 6 (HDAC6): a link between acetylation and the ubiquitination signaling pathways. HDAC6 is primarily localized in the cytoplasm, where it interacts with SIRT2 and with two ubiquitin pathway proteins, phospholipase A2 activating protein and p97/VCP. HDAC6 targets tubulin and possibly other cytoplasmic acetylated proteins for deacetylation. The ZnF-UBP domain of HDAC6 interacts specifically with ubiquitinated (Ub) proteins. This interaction could provide a functional link between the deacetylation of target protein(s) and their modification by ubiquitination. HDAC6 also shuttles between the nucleus and the cytoplasm. Nuclear targets have not been identified, but the known enzymatic activity of HDAC6 on histones suggests that it could participate in the regulation of nuclear gene expression by controlling histone acetylation in that compartment as well.

$\alpha$-tubulin both in vitro and in vivo [56]. Both HDAC6 and SIRT2 interact and co-localize in the cytoplasm with the microtubule network (Fig. 4) [56]. Suppression of either HDAC6 or SIRT2 mediated by siRNA leads to a relative hyperacetylation of $\alpha$-tubulin acetylation in vivo $[56,87,88]$. The identification of specific enzymes mediating tubulin deacetylation should allow further work to proceed on the role of this modification in microtubule dynamics and functions.

\section{Future questions}

The purification, cloning and characterization of class II HDACs have provided important insights into how chromatin controls transcription and have revealed unexpected biological functions for acetylation. The past two years have seen the elucidation of discrete biological pathways in which class II HDACs play crucial regulatory roles. Whereas class IIa HDACs are frequently considered functionally redundant, it is likely that further study will reveal discrete biological functions specific for each family member and its splice variants. Future studies should focus on determining the relative contribution of each HDAC in distinct cell types. By virtue of their ability to interact with distinct transcription factors that each bind at multiple sites in the genome, HDACs are likely to be master regulators that control differentiation pathways. Emphasis should therefore be placed on identifying the subsets of genes controlled by individual HDACs in various cell types. Genome-wide mapping of the histone deacetylation targets of the yeast HDAC proteins (RPD3, HDA1, Sir2, Hos1/3 and Hos2) has shown a clear division of labor between these HDACs [90]. For example, of
815 genomic regions targeted by RPD3 and of 647 regions targeted by HDA1, only $139(\sim 10 \%)$ are targeted by both HDACs. Whereas most published work has focused on nuclear HDACs acting on histone proteins, nonhistone proteins are probable targets for these enzymes, as recently illustrated for HDAC6 and tubulin.

A full understanding of the biology of class II HDACs will also require a detailed analysis of how these factors function biochemically as repressors of transcription. Because HDACs have proved difficult to purify as enzymatically active proteins, little is known about their enzymatic specificity. It will be important to determine which histones in the nucleosome and which lysine residues in each histone are deacetylated by individual HDACs. Since most HDACs reside in multiprotein complexes containing several HDACs, the potential contribution of different HDACs to enzymatic specificity will need to be addressed.

Finally, the identification of HDAC6 and SIRT2 as part of a single complex with tubulin deacetylase activity raises many new questions. The involvement of the HDAC6/SIRT2 complex in both tubulin deacetylation and protein ubiquitination suggests the existence of cross-talk between these two processes, a dialogue that is likely to be repeated in many proteins that are regulated by ubiquitination. A focus on in vivo experiments, including the development of a mouse knockout for each HDAC, together with a renewed effort to characterize the enzymatic activities of these proteins, will allow these important questions to be addressed.

\section{Acknowledgements}

We thank John Carroll and Jack Hull for graphics, and Stephen Ordway and Gary Howard for editorial assistance. We thank colleagues for sharing unpublished observations and apologize to those whose work could not be cited due to space constraints. Our work is supported by the NIH, The Sandler Foundation and the J. David Gladstone Institutes.

\section{References}

1 Wolffe, A.P. et al. (2000) Review: chromatin structural features and targets that regulate transcription. J. Struct. Biol. 129, 102-122

2 Cheung, W.L. et al. (2000) Acetylation and chromosomal functions. Curr. Opin. Cell Biol. 12, 326-333

3 Fischle, W. et al. (2001) The emerging role of class II histone deacetylases. Biochem. Cell Biol. 79, 337-348

4 Fischle, W. et al. (1999) A new family of human histone deacetylases related to Saccharomyces cerevisiae HDA1p. J. Biol. Chem. 274, $11713-11720$

5 Grozinger, C.M. et al. (1999) Three proteins define a class of human histone deacetylases related to yeast Hda1p. Proc. Natl. Acad. Sci. U. S. A. $96,4868-4873$

6 Miska, E.A. et al. (1999) HDAC4 deacetylase associates with and represses the MEF2 transcription factor. EMBO J. 18, 5099-5107

7 Wang, A.H. et al. (1999) HDAC4, a human histone deacetylase related to yeast HDA1, is a transcriptional co-repressor. Mol. Cell. Biol. 19, $7816-7827$

8 Tong, J.J. et al. (2002) Identification of HDAC10, a novel class II human histone deacetylase containing a leucine-rich domain. Nucleic Acids Res. 30, 1114-1123

9 Guardiola, A.R. et al. (2002) Molecular cloning and characterization of a novel histone deacetylase HDAC10. J. Biol. Chem. 277, 3350-3356

10 Fischer, D.D. et al. (2002) Isolation and characterization of a novel class II histone deacetylase, HDAC10. J. Biol. Chem. 277, 6656-6666

11 Gao, L. et al. (2002) Cloning and functional characterization of HDAC11, a novel member of the human histone deacetylase family. J. Biol. Chem. 277, 25748-25755 
12 Kao, H.Y. et al. (2002) Isolation and characterization of mammalian HDAC10, a novel histone deacetylase. J. Biol. Chem. 277, 187-193

13 Verdel, A. et al. (1999) Identification of a new family of higher eukaryotic histone deacetylases. Coordinate expression of differentiation-dependent chromatin modifiers. J. Biol. Chem. 274, $2440-2445$

14 Zhou, X. et al. (2000) Identification of a transcriptional repressor related to the noncatalytic domain of histone deacetylases 4 and 5 . Proc. Natl. Acad. Sci. U. S. A. 97, 1056-1061

15 Zhou, X. et al. (2001) Cloning and characterization of a histone deacetylase, HDAC9. Proc. Natl. Acad. Sci. U. S. A. 98, 10572-10577

16 Fischle, W. et al. (2001) Human HDAC7 histone deacetylase activity is associated with HDAC3 in vivo. J. Biol. Chem. 276, 35826-35835

17 Kao, H.Y. et al. (2000) Isolation of a novel histone deacetylase reveals that class I and class II deacetylases promote SMRT-mediated repression. Genes Dev. 14, 55-66

18 Chinnadurai, G. (2002) CtBP, an unconventional transcriptional co-repressor in development and oncogenesis. Mol. Cell 9, 213-224

19 Zhang, C.L. et al. (2001) Association of $\mathrm{COOH}$-terminal-binding protein (CtBP) and MEF2-interacting transcription repressor (MITR) contributes to transcriptional repression of the MEF2 transcription factor. J. Biol. Chem. 276, 35-39

20 Grozinger, C.M. et al. (2000) Regulation of histone deacetylase 4 and 5 and transcriptional activity by 14-3-3-dependent cellular localization. Proc. Natl. Acad. Sci. U. S. A. 97, 7835-7840

21 Wang, A.H. et al. (2000) Regulation of histone deacetylase 4 by binding of 14-3-3 proteins. Mol. Cell. Biol. 20, 6904-6912

22 McKinsey, T.A. et al. (2000) Activation of the myocyte enhancer factor-2 transcription factor by calcium/calmodulin-dependent protein kinase-stimulated binding of 14-3-3 to histone deacetylase 5. Proc. Natl. Acad. Sci. U. S. A. 97, 14400-14405

23 Kao, H.Y. et al. (2001) Mechanism for nucleocytoplasmic shuttling of histone deacetylase 7. J. Biol. Chem. 276, 47496-47507

24 Zhang, C.L. et al. (2001) The transcriptional co-repressor MITR is a signal-responsive inhibitor of myogenesis. Proc. Natl. Acad. Sci. U. S. A. 98, 7354-7359

25 Youn, H-D. et al. (2000) Calcium regulates transcriptional repression of myocyte enhancer factor 2 by histone deacetylase 4. J. Biol. Chem. $275,22563-22567$

26 McKinsey, T.A. et al. (2000) Signal-dependent nuclear export of a histone deacetylase regulates muscle differentiation. Nature 408 , $106-111$

27 Kerckaert, J.P. et al. (1993) LAZ3, a novel zinc-finger encoding gene, is disrupted by recurring chromosome 3q27 translocations in human lymphomas. Nat. Genet. 5, 66-70

28 Ye, B.H. et al. (1993) Alterations of a zinc finger-encoding gene, BCL-6, in diffuse large-cell lymphoma. Science 262, 747-750

29 Dhordain, P. et al. (1997) Co-repressor SMRT binds the BTB/POZ repressing domain of the LAZ3/BCL6 oncoprotein. Proc. Natl. Acad. Sci. U. S. A. 94, 10762-10767

30 Wong, C.W. et al. (1998) Components of the SMRT co-repressor complex exhibit distinctive interactions with the $\mathrm{POZ}$ domain oncoproteins PLZF, PLZF-RAR $\alpha$, and BCL-6. J. Biol. Chem. 273, 27695-27702

31 Huynh, K.D. et al. (1998) The BCL-6 POZ domain and other POZ domains interact with the co-repressors N-CoR and SMRT. Oncogene $17,2473-2484$

32 Huynh, K.D. et al. (2000) BCoR, a novel co-repressor involved in BCL-6 repression. Genes Dev. 14, 1810-1823

33 Shaffer, A.L. et al. (2000) BCL-6 represses genes that function in lymphocyte differentiation, inflammation, and cell cycle control. Immunity 13, 199-212

34 Lemercier, C. et al. (2002) Class II histone deacetylases are directly recruited by BCL6 transcriptional repressor. J. Biol. Chem. 277 , 22045-22052

35 Zhang, C.L. et al. (2002) Association of class II histone deacetylases associate with heterochromatin protein 1: a potential role for histone methylation in the control of muscle differentiation. Mol. Cell. Biol. 22 , $7302-7312$

36 Kirsh, O. et al. (2002) The SUMO E3 ligase RanBP2 promotes modification of the HDAC4 deacetylase. EMBO J. 21, 2682-2691

37 David, G. et al. (2002) SUMO-1 modification of histone deacetylase 1
(HDAC1) modulates its biological activities. J. Biol. Chem. 277, 23658-23663

38 Dressel, U. et al. (2001) A dynamic role for HDAC7 in MEF2-mediated muscle differentiation. J. Biol. Chem. 276, 17007-17013

39 Zhao, X. et al. (2001) The modular nature of histone deacetylase HDAC4 confers phosphorylation-dependent intracellular trafficking. J. Biol. Chem. 276, 35042-35048

40 Miska, E.A. et al. (2001) Differential localization of HDAC4 orchestrates muscle differentiation. Nucleic Acids Res. 29, 3439-3447

41 Muslin, A.J. et al. (2000) 14-3-3 proteins: regulation of subcellular localization by molecular interference. Cell. Signal. 12, 703-709

42 Zhou, X. et al. (2000) Histone deacetylase 4 associates with extracellular signal-regulated kinases 1 and 2, and its cellular localization is regulated by oncogenic Ras. Proc. Natl. Acad. Sci. U. S. A. $97,14329-14333$

43 McKinsey, T.A. et al. (2000) Signal-dependent nuclear export of a histone deacetylase regulates muscle diferentiation. Nature 408, 106-111

44 McKinsey, T.A. et al. (2001) Identification of a signal-responsive nuclear export sequence in class II histone deacetylases. Mol. Cell. Biol. 21, 6312-6321

45 Zhang, C.L. et al. (2002) Class II histone deacetylases act as signalresponsive repressors of cardiac hypertrophy. Cell 110, 479-488

46 Wang, A.H. et al. (2001) Histone deacetylase 4 possesses intrinsic nuclear import and export signals. Mol. Cell. Biol. 21, 5992-6005

$47 \mathrm{Lu}$, J. et al. (2000) Regulation of skeletal myogenesis by association of the MEF2 transcription factor with class II histone deacetylases. Mol. Cell 6, 233-244

48 Fischle, W. et al. (2002) Enzymatic activity associated with class II HDACs is dependent on a multiprotein complex containing HDAC3 and SMRT/N-CoR. Mol. Cell 9, 45-57

49 Huang, E.Y. et al. (2000) Nuclear receptor co-repressors partner with class II histone deacetylases in a Sin3-independent repression pathway. Genes Dev. 14, 45-54

50 Downes, M. et al. (2000) Identification of a nuclear domain with deacetylase activity. Proc. Natl. Acad. Sci. U. S. A. 97, 10330-10335

51 Aranda, A. et al. (2001) Nuclear hormone receptors and gene expression. Physiol. Rev. 81, 1269-1304

$52 \mathrm{Li}$, J. et al. (2000) Both co-repressor proteins SMRT and N-CoR exist in large protein complexes containing HDAC3. EMBO J. 19, 4342-4350

53 Guenther, M.G. et al. (2000) A core SMRT co-repressor complex containing HDAC3 and TBL1, a WD40-repeat protein linked to deafness. Genes Dev. 14, 1048-1057

54 Zhang, J. et al. (2002) The N-CoR-HDAC3 nuclear receptor co-repressor complex inhibits the JNK pathway through the integral subunit GPS2. Mol. Cell 9, 611-623

55 Pijnappel, W.W. et al. (2001) The S. cerevisiae SET3 complex includes two histone deacetylases, Hos2 and Hst1, and is a meiotic-specific repressor of the sporulation gene program. Genes Dev. 15, 2991-3004

56 North, B.J. et al. (2003) The human Sir2 ortholog, hSIRT2, is an $\mathrm{NAD}^{+}$-dependent tubulin deacetylase. Mol. Cell 11, 437-444

57 Wen, Y.D. et al. (2000) The histone deacetylase-3 complex contains nuclear receptor co-repressors. Proc. Natl. Acad. Sci. U. S. A. 97, $7202-7207$

58 Guenther, M.G. et al. (2001) The SMRT and N-CoR co-repressors are activating cofactors for histone deacetylase 3. Mol. Cell. Biol. 21, 6091-6101

59 McKinsey, T.A. et al. (2002) MEF2: a calcium-dependent regulator of cell division, differentiation and death. Trends Biochem. Sci. 27, 40-47

60 Mao, Z. et al. (1999) Neuronal activity-dependent cell survival mediated by transcription factor MEF2. Science 286, 785-790

61 Mao, Z. et al. (1999) Calcineurin enhances MEF2 DNA binding activity in calcium-dependent survival of cerebellar granule neurons. J. Biol. Chem. 274, 31102-31107

62 Okamoto, S. et al. (2000) Antiapoptotic role of the p38 mitogenactivated protein kinase-myocyte enhancer factor 2 transcription factor pathway during neuronal differentiation. Proc. Natl. Acad. Sci. U. S. A. $97,7561-7566$

63 McKinsey, T.A. et al. (2001) Control of muscle development by dueling HATs and HDACs. Curr. Opin. Genet. Dev. 11, 497-504

$64 \mathrm{Lu}$, J. et al. (2000) Signal-dependent activation of the MEF2 transcription factor by dissociation from histone deacetylases. Proc. Natl. Acad. Sci. U. S. A. 97, 4070-4075 
65 Sparrow, D.B. et al. (1999) MEF-2 function is modified by a novel co-repressor, MITR. EMBO J. 18, 5085-5098

66 Lemercier, C. et al. (2000) mHDA1/HDAC5 histone deacetylase interacts with and represses MEF2A transcriptional activity. J. Biol. Chem. 275, 15594-15599

67 Milbrandt, J. (1988) Nerve growth factor induces a gene homologous to the glucocorticoid receptor gene. Neuron 1, 183-188

68 Hazel, T.G. et al. (1988) A gene inducible by serum growth factors encodes a member of the steroid and thyroid hormone receptor superfamily. Proc. Natl. Acad. Sci. U. S. A. 85, 8444-8448

69 Ryseck, R.P. et al. (1989) Structure, mapping and expression of a growth factor inducible gene encoding a putative nuclear hormonal binding receptor. EMBO J. 8, 3327-3335

70 Woronicz, J.D. et al. (1994) Requirement for the orphan steroid receptor Nur77 in apoptosis of T-cell hybridomas. Nature 367, 277-281

71 Calnan, B.J. et al. (1995) A role for the orphan steroid receptor Nur77 in apoptosis accompanying antigen-induced negative selection. Immunity 3, 273-282

72 Woronicz, J.D. et al. (1995) Regulation of the Nur77 orphan steroid receptor in activation-induced apoptosis. Mol. Cell. Biol. 15, 6364-6376

73 Blaeser, F. et al. (2000) $\mathrm{Ca}(2+)$-dependent gene expression mediated by MEF2 transcription factors. J. Biol. Chem. 275, 197-209

74 Youn, H.D. et al. (2000) Integration of calcineurin and MEF2 signals by the coactivator p300 during T-cell apoptosis. EMBO J. 19, 4323-4331

75 Kasler, H.G. et al. (2000) ERK5 is a novel type of mitogen-activated protein kinase containing a transcriptional activation domain. Mol. Cell. Biol. 20, 8382-8389

76 Youn, H.D. et al. (1999) Apoptosis of $\mathrm{T}$ cells mediated by $\mathrm{Ca} 2+$-induced release of the transcription factor MEF2. Science 286, 790-793

77 Youn, H.D. et al. (2000) Cabin1 represses MEF2-dependent Nur77 expression and $\mathrm{T}$ cell apoptosis by controlling association of histone deacetylases and acetylases with MEF2. Immunity 13, 85-94

78 Esau, C. et al. (2001) Deletion of calcineurin and myocyte enhancer factor 2 (MEF2) binding domain of Cabin1 results in enhanced cytokine gene expression in T cells. J. Exp. Med. 194, 1449-1459

79 Tovey, M.G. et al. (1978) Activation of latent Epstein-Barr virus by antibody to human IgM. Nature 276, 270-272

80 zur Hausen, H. et al. (1978) Persisting oncogenic herpesvirus induced by the tumour promotor TPA. Nature 272, 373-375

81 Liu, S. et al. (1997) Cyclosporin A-sensitive induction of the EpsteinBarr virus lytic switch is mediated via a novel pathway involving a MEF2 family member. EMBO J. 16, 143-153

82 Gruffat, H. et al. (2002) MEF2-mediated recruitment of class II HDAC at the EBV immediate early gene BZLF1 links latency and chromatin remodeling. EMBO Rep. 3, 141-146

83 Seigneurin-Berny, D. et al. (2001) Identification of components of the murine histone deacetylase 6 complex: link between acetylation and ubiquitination signaling pathways. Mol. Cell. Biol. 21, 8035-8044

84 Furumai, R. et al. (2001) Potent histone deacetylase inhibitors built from trichostatin A and cyclic tetrapeptide antibiotics including trapoxin. Proc. Natl. Acad. Sci. U. S. A. 98, 87-92

85 Verdel, A. et al. (2000) Active maintenance of mHDA2/mHDAC6 histone-deacetylase in the cytoplasm. Curr. Biol. 10, 747-749

86 Hook, S.S. et al. (2002) Histone deacetylase 6 binds polyubiquitin through its zinc finger (PAZ domain) and copurifies with deubiquitinating enzymes. Proc. Natl. Acad. Sci. U. S. A. 99, 13425-13430

87 Hubbert, C. et al. (2002) HDAC6 is a microtubule-associated deacetylase. Nature 417, 455-458

88 Matsuyama, A. et al. (2002) In vivo destabilization of dynamic microtubules by HDAC6-mediated deacetylation. EMBO J. 21, 6820-6821

89 Piperno, G. et al. (1987) Microtubules containing acetylated $\alpha$-tubulin in mammalian cells in culture. J. Cell Biol. 104, 289-302

90 Robyr, D. et al. (2002) Microarray deacetylation maps determine genome-wide functions for yeast histone deacetylases. Cell 109, 437-446 